Supporting Information

\title{
Three-Dimensional Self-Healable Touch Sensing Artificial Skin Device
}

Sulbin Park, Byeong-Gwang Shin, Seongwan Jang and Kyeongwoon Chung*

3D Printing Materials Center, Korea Institute of Materials Science (KIMS), Changwon, 51508, South Korea

*Correspondence to: kychung@kims.re.kr 


\section{Materials and Experimental Procedures}

Materials Acrylic acid (Tokyo chemical industry), $\mathrm{FeCl}_{3}$ (Merck), poly(ethylene glycol) diacrylate (PEGDA, $\mathrm{M}_{\mathrm{n}} \sim 250$, Merck), $\mathrm{NaCl}$ (Samchun chemicals) and ammonium persulfate (APS, Samchun chemicals) were purchased and used without further purification.

Preparation of Ion-Conductive Self-healing Hydrogel The pre-gel solution was prepared by mixing of acrylic acid $(1.5 \mathrm{M}), \mathrm{NaCl}(0.1 \mathrm{M})$, PEGDA $(7.5 \mathrm{mM})$, and $\mathrm{FeCl}_{3}(22.5 \mathrm{mM})$ in deionized water at room temperature. The solution was degassed with Argon bubbling for 30 minutes. Subsequently, 0.03 M of APS, as an initiator for free radical polymerization, was added to the solution and stored at $50{ }^{\circ} \mathrm{C}$ for 2 hours. The synthesized ion-conductive selfhealing hydrogel was further kept overnight at room temperature and rinsed with $0.1 \mathrm{M} \mathrm{NaCl}$ solution to remove residual monomers.

Rheology Characterization Rheological measurement of the hydrogel was carried out with a rheometer (MCR 302, Anton Paar) at room temperature. An oscillatory shear is applied to the hydrogel located between two parallel plates for time dependent self-healing experiment and frequency sweep. The time dependent self-healing experiment is conducted by applying shear strain of $5 \%$ for $120 \mathrm{sec}$, followed by high shear strain of $200 \%$ for $30 \mathrm{sec}$, and released back to $5 \%$ for $600 \mathrm{sec}$, while measuring shear storage modulus (G'), shear loss modulus (G'), and complex viscosity $\left(\eta^{*}\right)$. A frequency sweep of hydrogel was conducted in the frequency range of $0.1 \sim 100 \mathrm{rad} / \mathrm{s}$ at the fixed shear strain of $5 \%$.

FTIR and Raman spectroscopy Fourier transform infrared spectroscopy (FTIR) of the ionconductive self-healing hydrogel was conducted with attenuated total reflection (ATR) mode using Nicolet iS10, Thermo scientific. The Raman spectroscopy of the hydrogel was conducted 
using an optical Raman system (NS220, Nanoscope Systems, Inc.). The laser power, wavelength, and exposure time were $6 \mathrm{~mW}, 633 \mathrm{~nm}$, and $3 \mathrm{sec}$, respectively.

Ion Conductivity Measurement Ion conductivity of the hydrogel was characterized using broadband dielectric/impedance spectrometer (Concept 40, Novocontrol technologies). The ion-conductive self-healing hydrogel is sandwiched between two electrodes, and the electrode dimension and gap between electrodes are measured and applied in software for ion conductivity calculation. The ion conductivity of the hydrogel is measured under frequency variation from $10^{7} \mathrm{~Hz}$ to $1 \mathrm{~Hz}$ of $0.1 \mathrm{~V}$ alternating current (AC).

3D Printing of Ion-Conductive Self-healing Hydrogel The hydrogel was moved into a syringe and centrifuged for 2 4 minutes at 2000rpm for air bubble removal, and the syringe was installed in the extrusion-based 3D printers (SM200SX-3A, ML-5000XII-CTR, Musashi and a custom made 3D printer, $\mathrm{K}$ Labs). Metal nozzles used in all experiments were in size of inner diameter ca. $0.60 \mathrm{~mm}$. To secure the quality of printed hydrogel, the printing conditions are set as printing speed of $0.5 \sim 5 \mathrm{~mm} / \mathrm{s}$ and extrusion pressure of $150 \sim 400 \mathrm{kPa}$. In case of three-dimensional ring-shaped hydrogels, printing speed of $0.5 \mathrm{~mm} / \mathrm{s}$ and extrusion pressure of $170 \mathrm{kPa}$ were applied for 3D Printing. In case of three-dimensional fingertip-shaped hydrogels, printing speed of $2.0 \mathrm{~mm} / \mathrm{s}$ and extrusion pressure of $300 \mathrm{kPa}$ were applied for 3D Printing. The printed hydrogel is applied in the tactile sensing application without further postprocessing.

Tactile Sensing Demonstration The human finger touch sensing setups of ion-conductive self-healing hydrogel devices were built with a function generator (WF1946B, NF) which applies same phase $\mathrm{AC}(100 \mathrm{kHz}, 0.5 \mathrm{~V})$ to all electrodes that connected to the hydrogel, and 
multimeters (289 true RMS multimeter, Fluke) that records the current changes when human finger touches the hydrogel artificial skin. Three different types of devices were demonstrated; a bulk hydrogel device, a ring-shaped hydrogel device, and a fingertip-shaped hydrogel device. As-prepared hydrogel (for bulk hydrogel device) and as-3D-printed hydrogels (for threedimensional ring-shaped and fingertip-shaped hydrogel devices) are utilized as an active material in the touch sensing devices without further treatment or post-processing. In case of the 3D printed ring-shaped and fingertip-shaped hydrogels, the printed hydrogels were worn over plastic finger to prepare three-dimensional artificial skin devices. The hydrogel is simply connected to electodes to complete device preparation. There were two electrodes and multimeters connected to the hydrogel in case of the bulk hydrogel tactile sensing device (Figure S5) and ring-shaped hydrogel artificial skin device (Figure 3) to measure current signal variation and define exact human finger contact location. On the other hand, three electrodes and multimeters are installed in $120^{\circ}$ apart in case of finger-tip shaped hydrogel artificial skin device (Figure 4, Figure S9 and S10) for current signal detection as well as mapping of the exact touch location. 


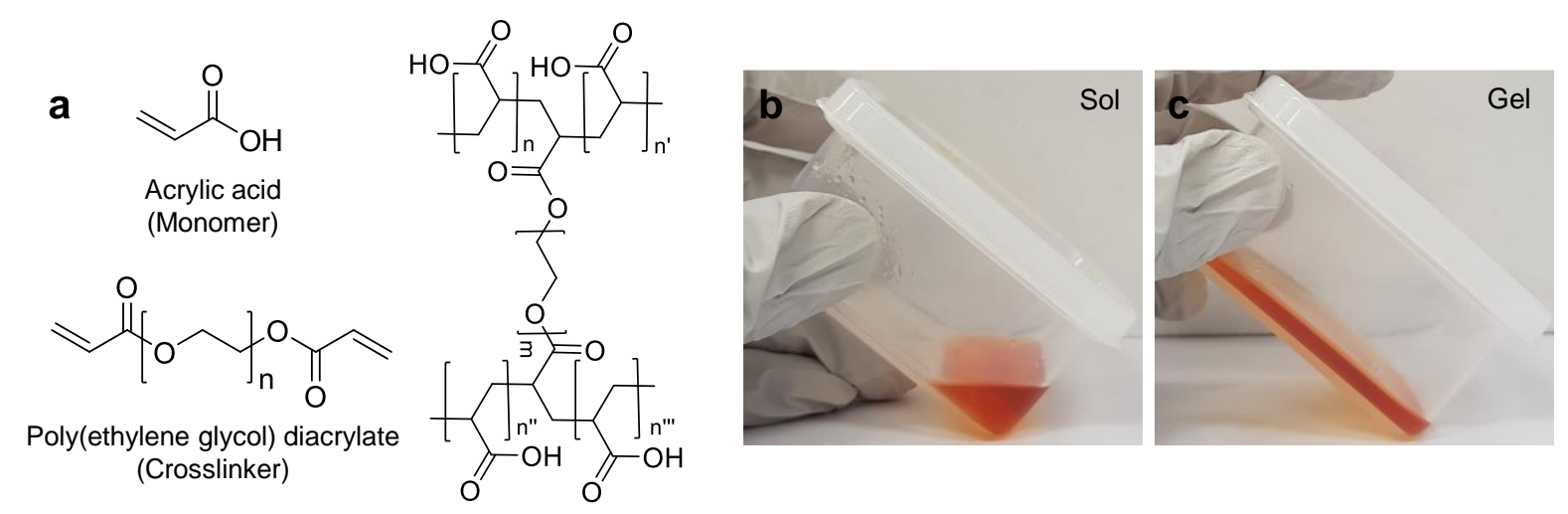

Figure S1. Design and Gelation of ion-conductive self-healing hydrogel. a, Chemical structure of monomer, crosslinker, and polymer network for the hydrogel. b and c, photographs of pre-gel solution (b) and synthesized hydrogel (c) under room light.
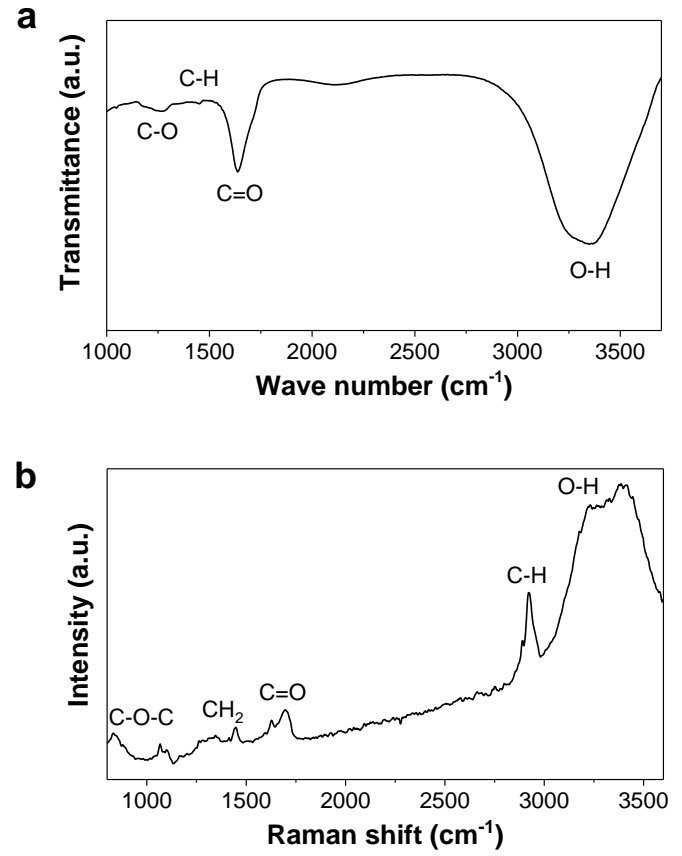

Figure S2. Characterization of polymer network in the ion-conductive self-healing hydrogel. a, Fourier transform infrared (FTIR) spectra. b, Raman spectra. 
a

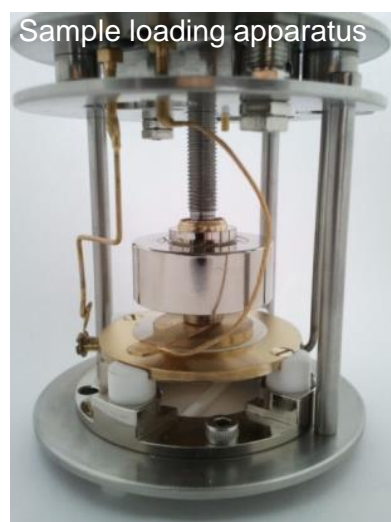

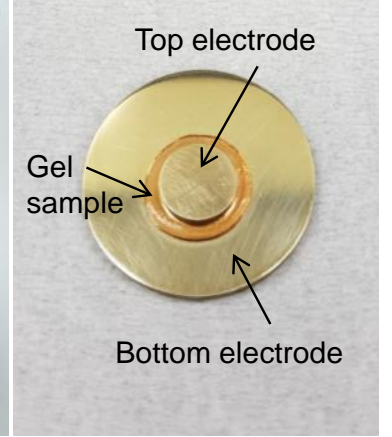

b

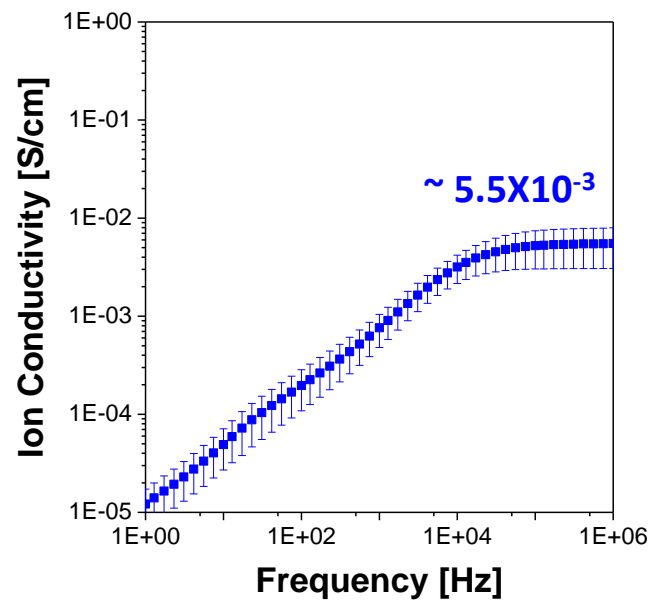

Figure S3. Ion conductivity measurement of the ion-conductive self-healing hydrogel. a,

Sample loading setup for the ion conductivity measurement using broadband dielectric/impedance spectrometer. b, Ion conductivity of the hydrogel. From the plateau in high frequency area, the ion conductivity of the hydrogel is characterized as ca. $5.5 \times 10^{-3} \mathrm{~S} / \mathrm{cm}$. 


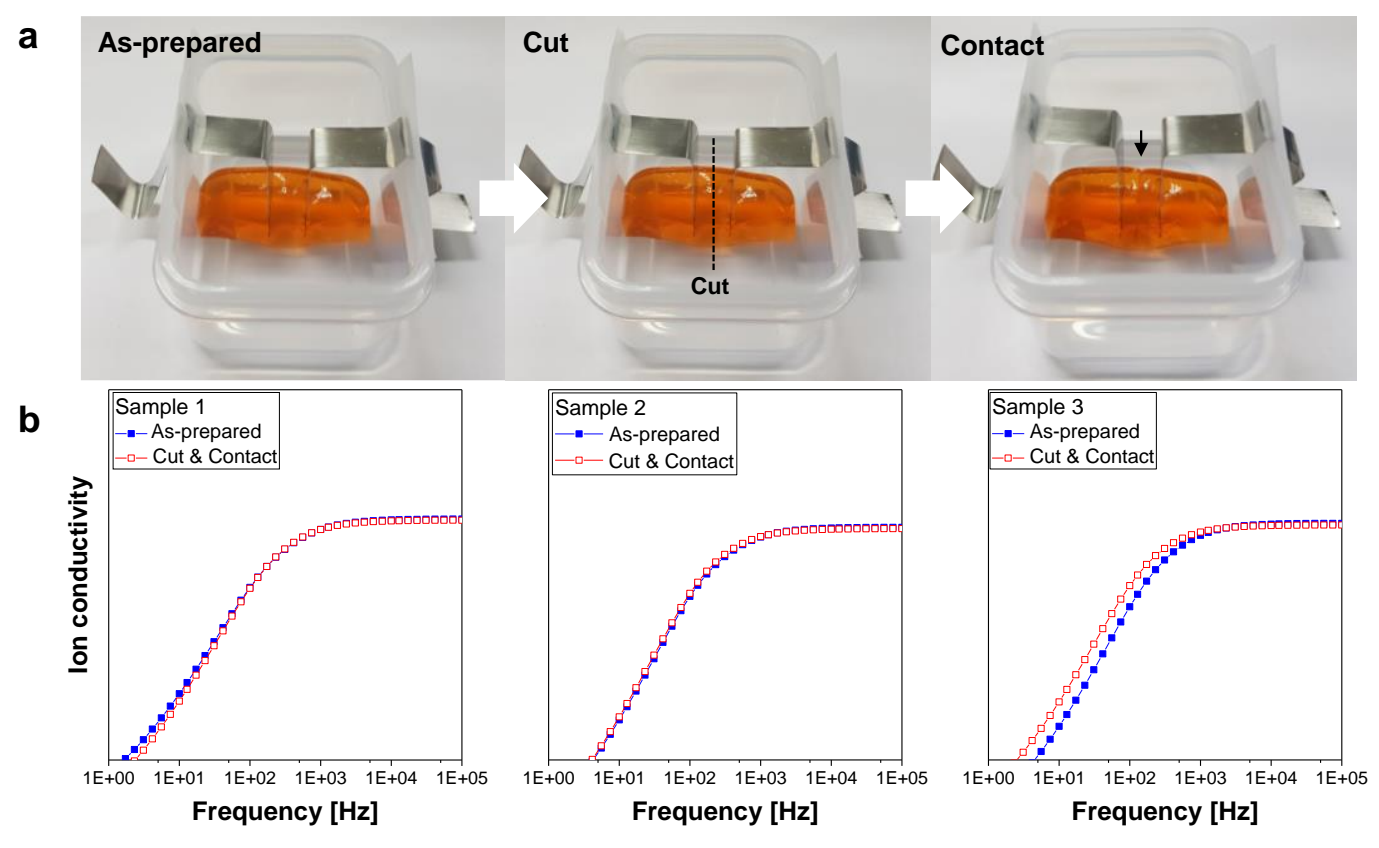

Figure S4. Ion conductivity recovery of the ion-conductive self-healing hydrogel before and after cut-and-contact. a, Experimental setup to characterize ion conductivity recovery. The ion conductivity of as-prepared hydrogel is measured, followed by cut of the hydrogel sample into two pieces. The two pieces of the hydrogel are contacted each other and healed for $10 \mathrm{~min}$, and repeated frequency sweep for ion conductivity measurement. b, Ion conductivity of three hydrogel samples before and after cut-and-contact. From the plateau in high frequency area, the hydrogel samples show average ion conductivity recovery of $97 \%$ in $10 \mathrm{~min}$. 

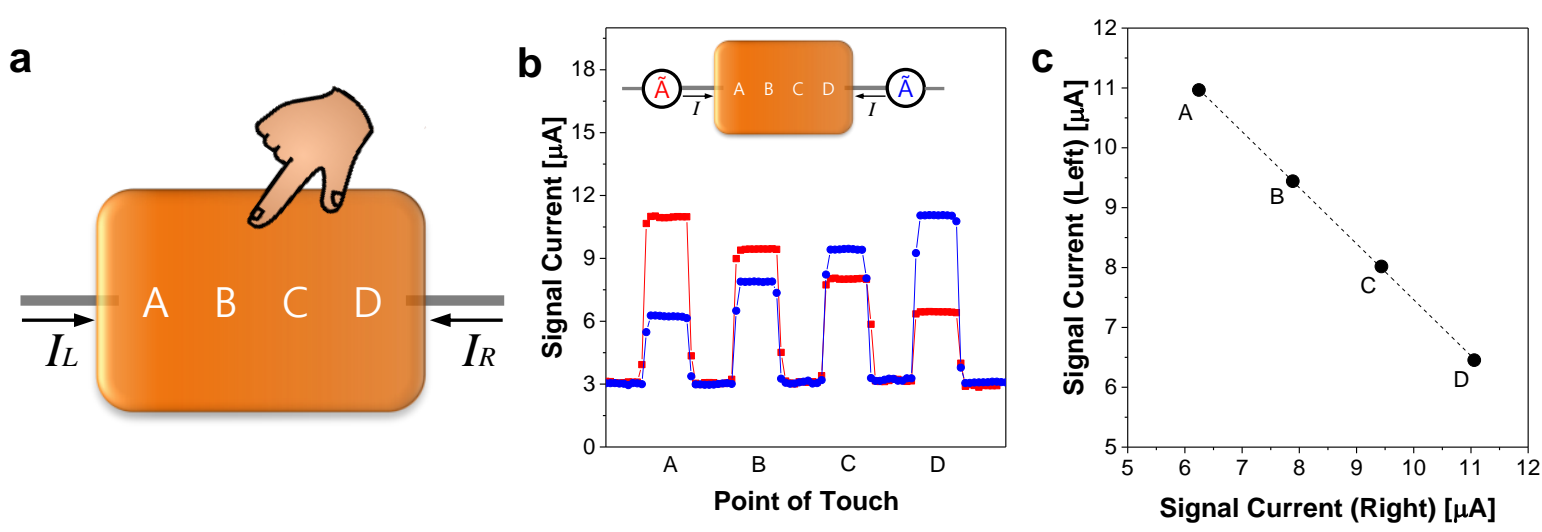

Figure S5. Touch sensing characteristics of the two-dimensional bulk ion-conductive selfhealing hydrogel. a, Schematic illustration of a touch sensing device based on a block of the ion-conductive self-healing hydrogel. The touch locations, A, B, C, D, and two electrodes on each side of the hydrogel are $10 \mathrm{~mm}$ apart each other. Alternating current (AC) of same phase is applied on the electrodes to build a uniform electrostatic field in the hydrogel block (100 $\mathrm{kHz}, 0.5 \mathrm{~V})$. b, Signal current generated by a human finger touch on the two-dimensional touch sensing device. The device shows large electronic signal contrast upon touch ( 3.6 times increased), and shows gradual change in output current inversely proportional to the distance from a touch position to the electrodes. c, two-dimensional plot of the signal currents from two electrodes. The current signals from touchpoints (A D) are aligned on a line with a slope of ca. -1 , supporting that the current signal is completely governed by the distance from a touchpoint to the electrodes. 

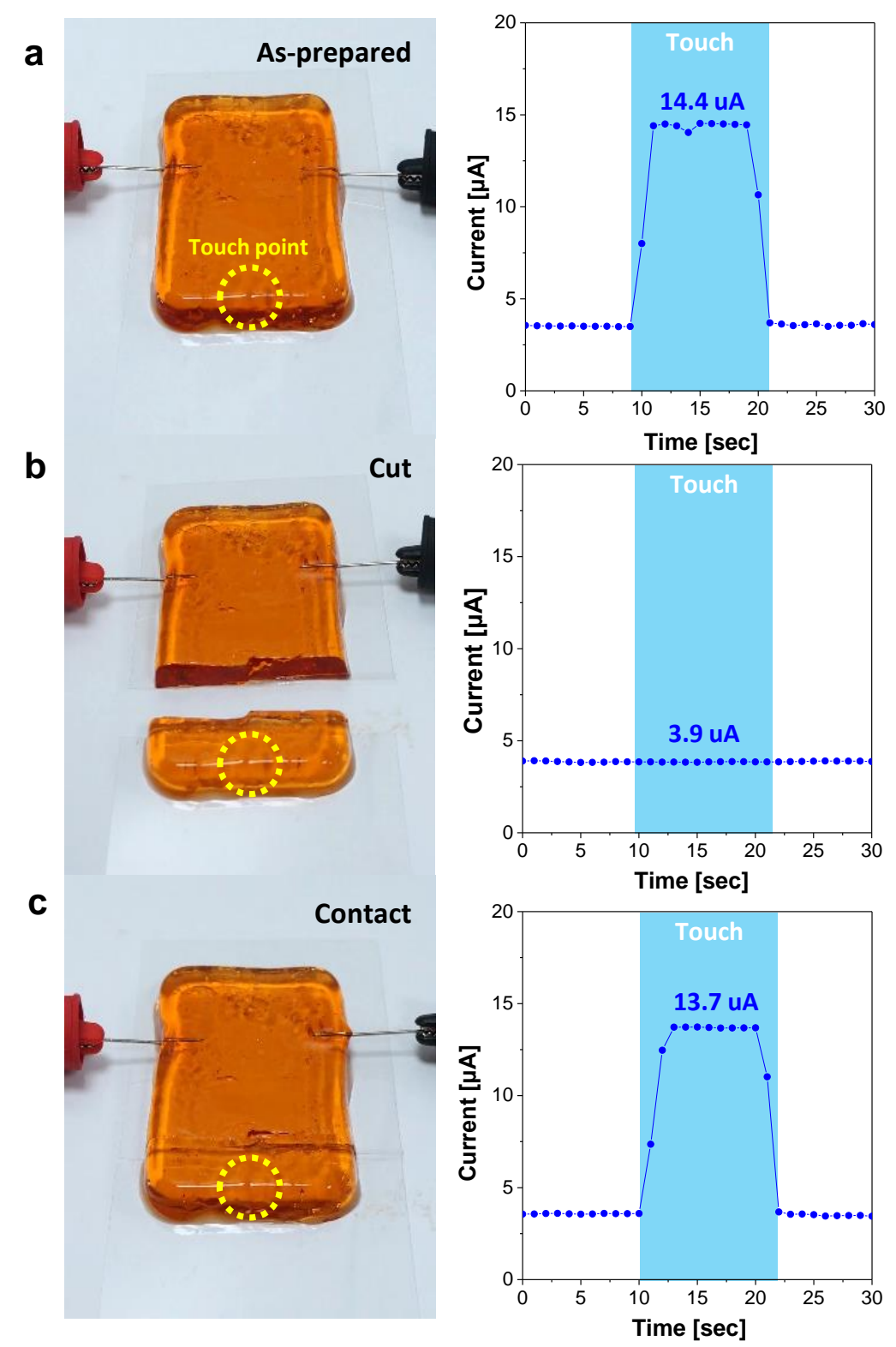

Figure S6. Sensing characteristic of the ion-conductive self-healing hydrogel under applied mechanical damage and self-healing process. a-c, Experimental setup and current signals generated upon a finger touch under cut-and-contact of the hydrogel. 
a

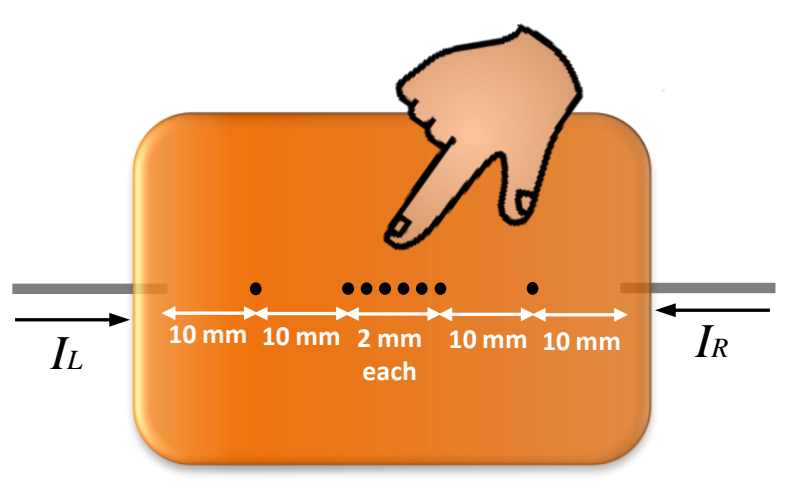

b

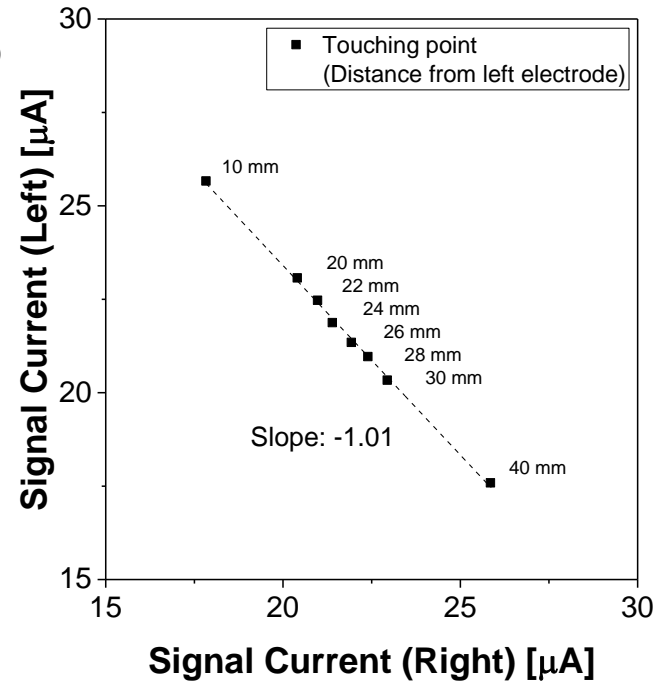

Figure S7. Characterization of spatial resolution of the touch sensing device. a, Schematic illustration of a touch sensing device. The touchpoints located in the middle of the device are $2 \mathrm{~mm}$ apart. b, two-dimensional plot of the signal currents from two electrodes. The current signals from each touchpoints are completely aligned on a line with a slope of -1 , which demonstrates capability to precisely locate the unknown touchpoints on the artificial skin device even in the case that the touchpoints are only $2 \mathrm{~mm}$ apart.
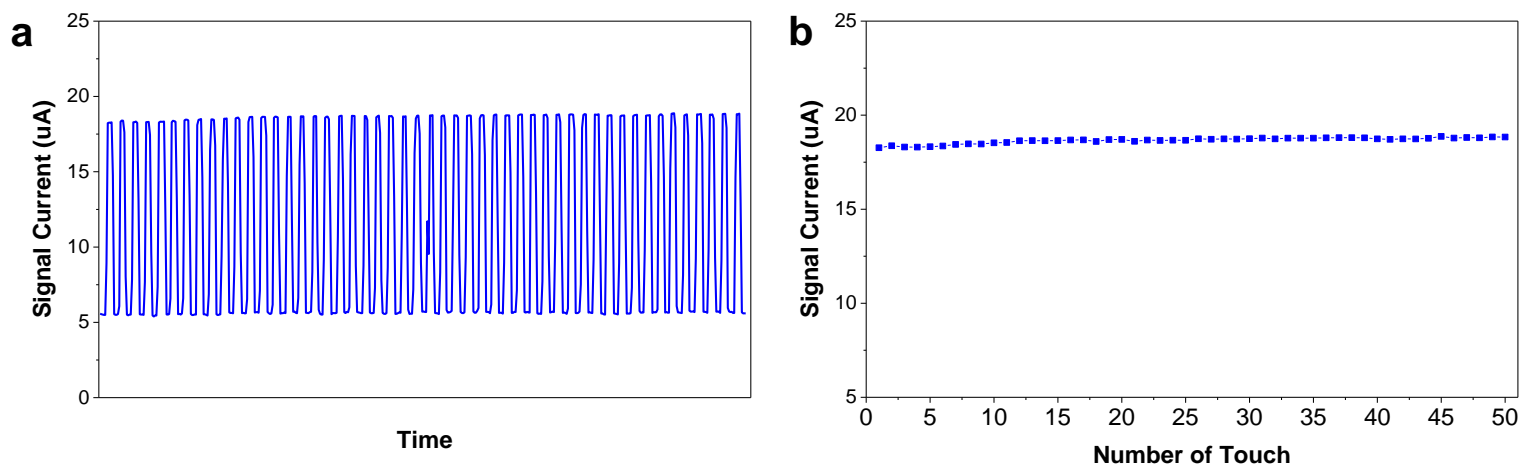

Figure S8. Touch sensing durability of the artificial skin device. a, Signal current generation upon fifty repeated touches. b, Signal current retaining during fifty repeated touches. 
a

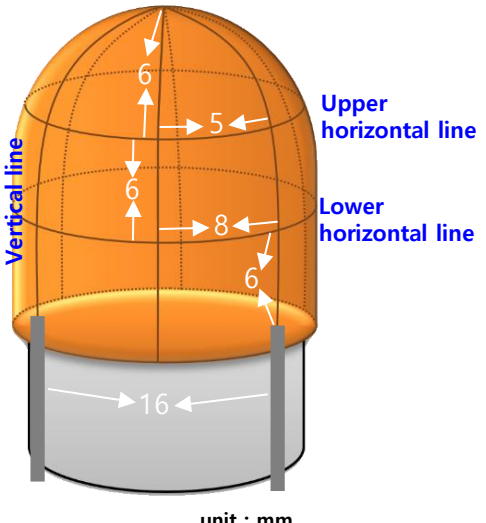

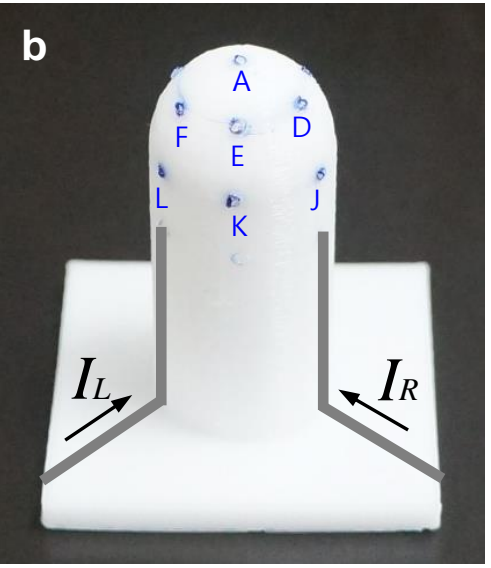

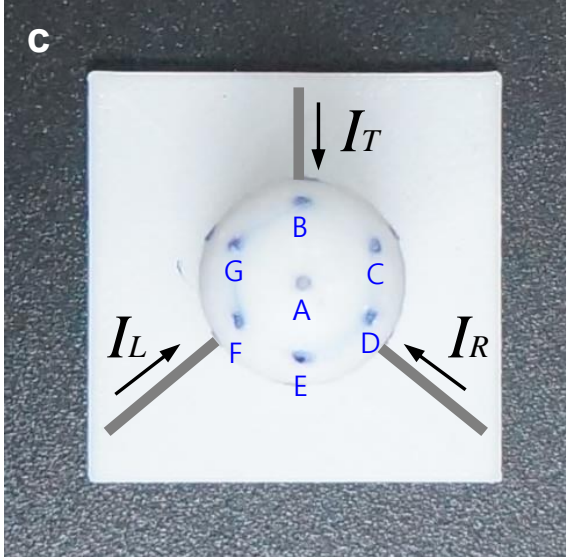

Figure S9. The spatial location of the touchpoints in the fingertip-shaped artificial skin device. a, Schematic illustration of distance (unit: $\mathrm{mm}$ ) between the touchpoints (lines). $\mathbf{b}$ and c, Side view (b) and top view (c) of the artificial plastic finger. The position of touchpoints and electrodes are marked in the figure.

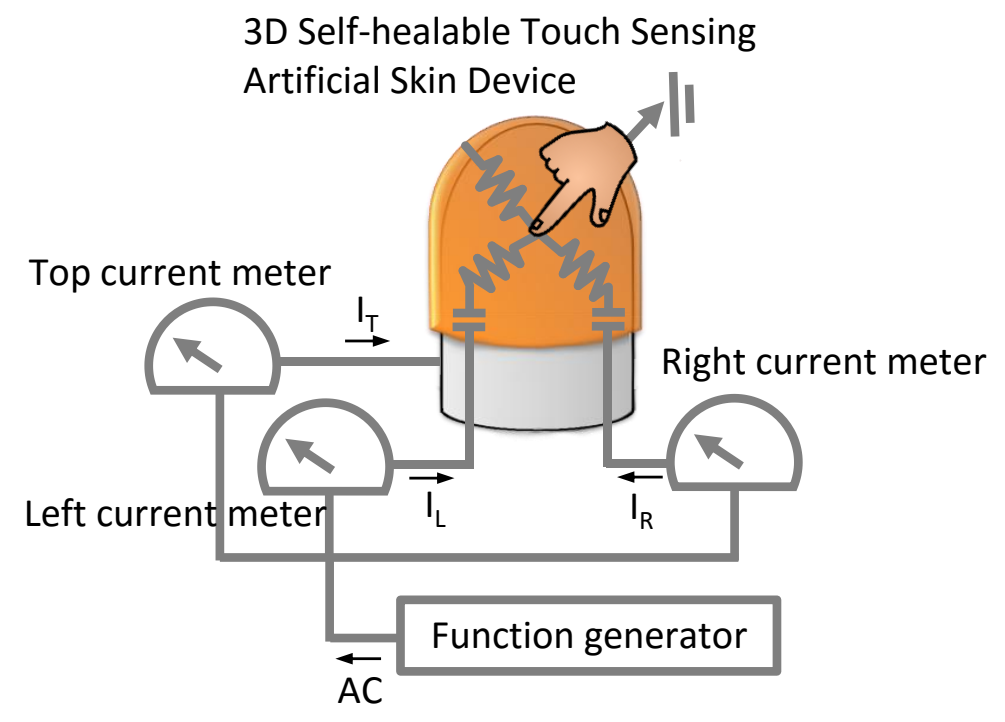

Figure S10. The circuit diagram of three-dimensional fingertip-shaped artificial skin device. Three electrodes (Top, Left, Right) are connected on the as-printed hydrogel. Same phase alternating current from function generator $(\mathrm{AC}, 100 \mathrm{kHz}, 0.5 \mathrm{~V})$ is applied on each electrode which builds a uniform electrostatic field in the hydrogel device. 